\title{
RAPID VARIATIONS OF Ha IN Be STARS
}

\author{
J. D. R. BAHNG* \\ Astronomy Dept., Northwestern University, Evanston, Ill., U.S.A.
}

\begin{abstract}
Photoelectric spectrum scans of Be stars were analysed to study the short-term variations of $\mathrm{H} \alpha$ emission strengths. In $\zeta$ Tau, $\alpha \mathrm{Col}$, PP Car, and $\delta$ Cen, definite variations of a few percent with time scales of 1 to 3 minutes were found. These variations do not exhibit any periodicity.
\end{abstract}

\section{Introduction}

Recently, several reports have been published in which rapid spectral changes in $\mathrm{Be}$ stars with time scales of a few minutes were observed. Profiles of hydrogen emission lines were found to vary in a time scale as short as one minute (Hutchings et al., 1971). Other studies (Bahng, 1971; McBeath, 1974; Sanyal, 1974) indicate variations in the total emission strengths of hydrogen lines with a time scale of one to ten minutes. The profile changes observed by Hutchings et al. appear to be accompanied by some changes in the total line strengths as well.

It is quite clear that these rapid changes cannot be explained in terms of the binary orbital motions (Delplace, 1970), a geometry connected with the asymmetric distribution of gases in a circular ring (Huang, 1972), or an elliptical ring (Huang, 1973). It is equally unlikely that they are related to oscillations or pulsations of the star or shell, since the observed time scale is much too short. As yet, no satisfactory theoretical models exist to explain these rapid phenomena. From the time scales involved, Mihalas (1974) concluded that they imply hydrodynamic interactions; a model proposed by Marlborough and Zamir (1975) may be relevant. On the other hand, some unknown radiative processes (see e.g. Prendergast and Spiegel, 1973) may be responsible for these changes.

It is extremely important to establish first the reality of these rapid changes. If these variations do indeed occur, then the following questions must be answered. (1) What are the precise time scales? (2) What is the nature of the variations? Are they periodic, recurring, or completely random flickering? (3) What is the amplitude or range of variations? And finally, (4) Are there any common characteristics in these, or any correlations with the known parameters of these stars?

For some time, a study has been underway to investigate these problems with regard to the total emission strengths of hydrogen lines in early-type stars.

\section{Observations}

Observations were made of four Be stars in December 1973 at Kitt Peak National Observatory, and of five Be stars in February 1974 at Cerro Tololo

\footnotetext{
* Visiting Astronomer, Kitt Peak National Observatory and Cerro Tololo Inter-American Observatory, which are operated by the Association of Universities for Research in Astronomy, Inc., under contract with the National Science Foundation.
} 
Inter-American Observatory. In both cases, a two-channel low-resolution photoelectric spectrum scanner was used at the Cassegrain focus of the $91-\mathrm{cm}$ telescope. The entrance apertures were $31^{\prime \prime}$ in diameter separated by $4^{\prime} .5$ for Kitt Peak observations, and $18^{\prime \prime}$ separated by $2^{\prime} .6$ for Cerro Tololo observations. The range of spectrum covered in each scan was $\lambda 6530-6600 \AA$ in the first order. The band pass as defined by the width of exit slit was $4 \AA$ for the Kitt Peak observations and $2 \AA$ for the Cerro Tololo observations. The step size, the interval between two successive data points in the spectrum, was $2 \AA$.

The observing procedures were similar to those employed in an earlier study (Bahng, 1975). That is, each program star was observed continuously for about two hours to obtain a time series of spectrum scans. The Kitt Peak scanner could not be used in the automatic mode, so that data recording on a magnetic tape and the commencement of the next scanning operation had to be initiated manually. For this reason, each scan in the time series observations for the northern Be stars could not be made at a precisely uniform time interval. A number of normal main sequence $B$ stars were also observed as comparison stars.

TABLE I

Variations of $\mathrm{H} \alpha$ equivalent widths - Northern stars

\begin{tabular}{llcccccc}
\hline Star & $\begin{array}{l}\text { Date }(\mathrm{UT}) \\
1973\end{array}$ & $\begin{array}{l}\text { Number } \\
\text { of scans }\end{array}$ & $\bar{W}(\AA)$ & $\sigma_{o}(\AA)$ & $\sigma_{p}(\AA)$ & $q=\sigma_{o} / \sigma_{p}$ & $\bar{q}$ \\
\hline 1 Per & Dec. 15 & 3 & 4.38 & 0.21 & 0.14 & 1.50 & \\
B2 V & Dec. 16 & 3 & 4.48 & 0.37 & 0.20 & 1.85 & \\
& Dec. 17 & 5 & 4.33 & 0.17 & 0.18 & 0.94 & 1.43 \\
& & & & & & & \\
121 Tau & Dec. 14 & 3 & 5.14 & 0.26 & 0.20 & 1.30 & \\
B3 V & Dec. 15 & 15 & 5.02 & 0.24 & 0.14 & 1.71 & \\
& Dec. 16 & 7 & 4.94 & 0.34 & 0.20 & 1.70 & \\
& & & & & & & 1.57 \\
\hline \multirow{2}{*}{ 48 Per } & Dec. 14 & 6 & -20.11 & 0.58 & 0.24 & 2.42 & \\
B3 Ve & Dec. 16 & 6 & -20.21 & 0.55 & 0.20 & 2.75 & \\
& Dec. 17 & 13 & -20.17 & 0.52 & 0.20 & 2.60 & \\
& & & & & & & \\
$\zeta$ Tau & Dec. 14 & 97 & -19.90 & 0.67 & 0.25 & 2.68 & \\
B2 IVp & Dec. 15 & 80 & -20.44 & 0.66 & 0.17 & 3.88 & \\
& Dec. 16 & 39 & -23.01 & 1.04 & 0.30 & 3.47 & \\
& Dec. 17 & 105 & -23.21 & 1.01 & 0.28 & 3.61 & \\
& & & & & & & 3.41 \\
$\nu$ Gem & Dec. 16 & 16 & -2.49 & 0.30 & 0.19 & 1.58 & \\
B7 IVe & Dec. 17 & 14 & -2.29 & 0.21 & 0.19 & 1.11 & 1.35 \\
$\kappa$ Dra & Dec. 15 & 35 & -13.13 & 0.28 & 0.16 & 1.75 & \\
B7 p & Dec. 16 & 25 & -12.89 & 0.39 & 0.17 & 2.29 & \\
& & & & & & & 2.02 \\
& & & & & & & \\
\hline
\end{tabular}


TABLE II

Variations of $\mathrm{H} \alpha$ equivalent widths - Southern stars

\begin{tabular}{|c|c|c|c|c|c|c|c|c|}
\hline Star & $\begin{array}{l}\text { Date (UT) } \\
1974\end{array}$ & $\begin{array}{l}\text { Number } \\
\text { of scans }\end{array}$ & $\Delta t_{(\mathrm{Sec})}^{\mathrm{a}}$ & $\begin{array}{l}\vec{W} \\
(\AA)\end{array}$ & $\begin{array}{c}\sigma_{o} \\
(\AA)\end{array}$ & $\begin{array}{r}\sigma_{p} \\
(\AA)\end{array}$ & $q=\sigma_{o} / \sigma_{p}$ & $\bar{q}$ \\
\hline $\begin{array}{l}\chi \mathrm{Car} \\
\mathrm{B} 2 \mathrm{IV}\end{array}$ & b & b & b & 3.01 & 0.18 & 0.16 & 1.13 & \\
\hline $\begin{array}{l}\sigma \text { Cen } \\
\text { B2 V }\end{array}$ & b & b & b & 3.62 & 0.14 & 0.15 & 0.93 & \\
\hline $\begin{array}{l}\varepsilon \text { Cen } \\
\text { B1 V }\end{array}$ & Feb. 7 & 52 & 67.5 & 2.95 & 0.20 & 0.16 & 1.25 & $1.10^{\mathrm{c}}$ \\
\hline \multirow{2}{*}{$\begin{array}{l}\alpha \mathrm{Col} \\
\mathrm{B} 8 \mathrm{Ve}\end{array}$} & Feb. 7 & 99 & 100.5 & -8.57 & 0.47 & 0.17 & 2.77 & \\
\hline & Feb. 9 & 68 & 100.5 & -8.48 & 0.60 & 0.18 & 3.33 & 3.05 \\
\hline $\begin{array}{l}\kappa \mathrm{CMa} \\
\mathrm{B} 2 \mathrm{Ve}\end{array}$ & Feb. 8 & 32 & 337.3 & -17.66 & 0.39 & 0.19 & 2.05 & 2.05 \\
\hline \multirow{2}{*}{$\begin{array}{l}\omega \mathrm{Car} \\
\text { B7 IV }\end{array}$} & Feb. 7 & 65 & 202.4 & -5.20 & 0.26 & 0.16 & 1.63 & \\
\hline & Feb. 9 & 66 & 202.4 & -5.17 & 0.25 & 0.16 & 1.56 & 1.60 \\
\hline \multirow[t]{2}{*}{$\begin{array}{l}\text { PP Car } \\
\text { B5 Ve }\end{array}$} & Feb. 8 & 75 & 202.4 & -30.24 & 0.61 & 0.22 & 2.77 & \\
\hline & Feb. 11 & 36 & 202.4 & -29.12 & 0.74 & 0.20 & 3.70 & 3.24 \\
\hline \multirow{2}{*}{$\begin{array}{l}\delta \text { Cen } \\
\text { B2?V?pe }\end{array}$} & Feb. 11 & 76 & 67.5 & -32.64 & 1.02 & 0.25 & 4.08 & \\
\hline & Feb. 11 & 45 & 67.5 & -32.75 & 0.75 & 0.25 & 3.00 & 3.54 \\
\hline
\end{tabular}

\footnotetext{
a Time interval between successive scans.

b Standard stars, 2 scahs each night.

Mean from 3 standard stars.
}

\section{Results}

Since the spectral resolution is too low, the line-profile study cannot be made. Nor is it possible to study the $\boldsymbol{V}$-and $\boldsymbol{R}$-component separately. Instead, only the equivalent widths of $\mathrm{H} \alpha, W$, were obtained from each scan. For each time series, the mean equivalent width, $\bar{W}$, and a quantity $q$, defined by $q=\sigma_{o} / \sigma_{p}$, were computed. Here $\sigma_{o}$ is the usual rms value of observed $W$ from $\bar{W}$, and $\sigma_{p}$ is the expected rms value computed on the assumption that the scatter is due to the photon-counting statistics alone. Comparison of the $q$ values for Be stars with those for normal B stars will serve as a basis for deciding whether the observed variations in $W$ are significant.

The results are summarized in Tables I and II. The individual time series are shown in Figures 1 through 5. In these figures, the larger error bars give $\pm \sigma_{o}$, and the smaller error bars $\pm \sigma_{p}$ from the mean. For the southern stars, power spectrum analyses were performed. The mean power spectra weighted according to the number of scans in each time series, are shown along with the corresponding time series. The solid lines are power spectra computed from the observed data, while the crosses indicate the power spectra when a sinusoidal trace signal with a semi-amplitude of $0.5 \AA$ was superimposed on the observed data. 


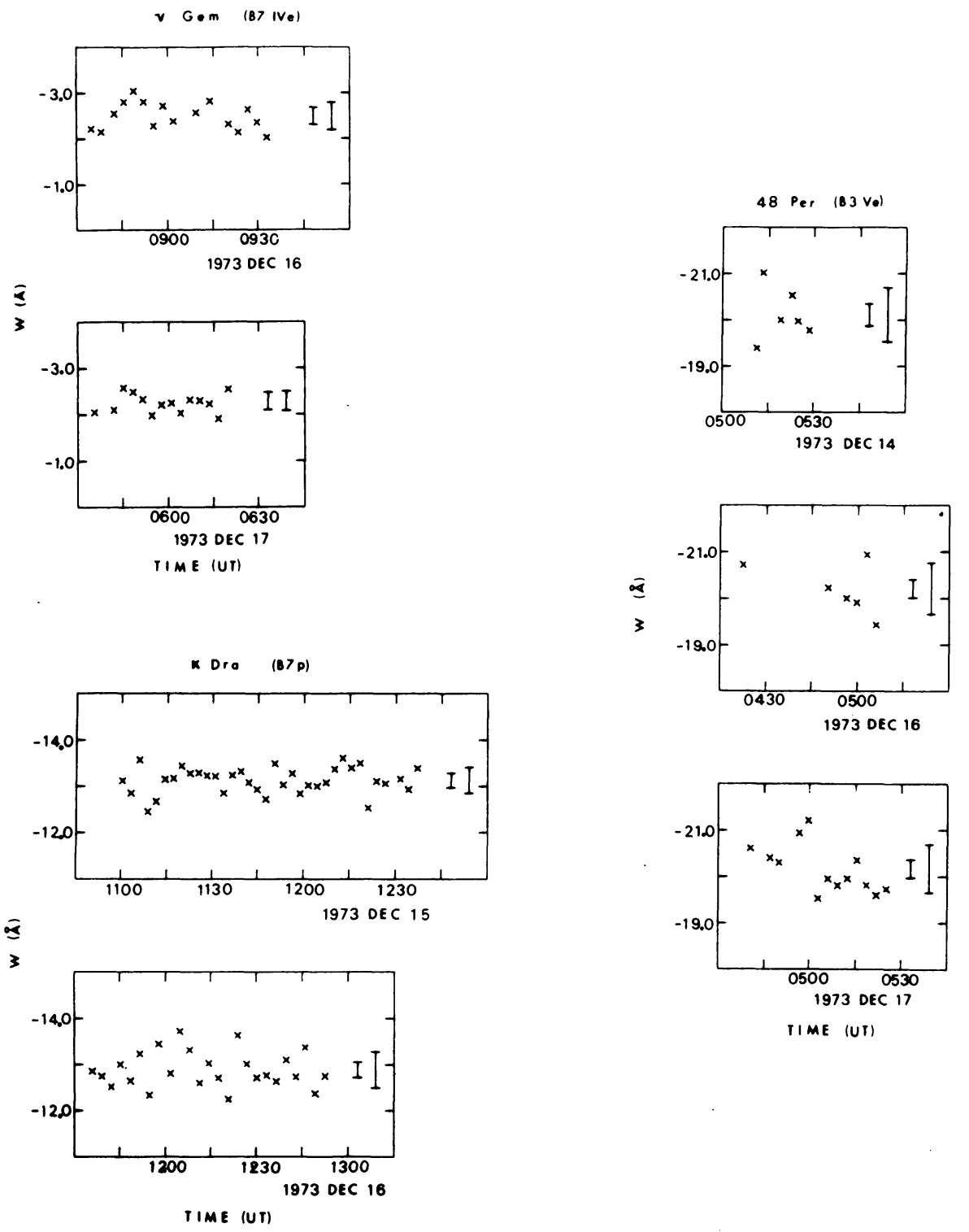

Fig. 1. H $\alpha$ equivalent width as a function of time for $\nu \mathrm{Gem}, \kappa$ Dra, and 48 Per. The error bars are observed rms (larger) and the computed photon rms (smaller).

\section{Discussion}

In order to assess the significance of variations in $W$, the values of $q$ for each Be star are compared with the $q$ for the comparison stars. For these stars, the values range from 0.93 to 1.85 with the mean of 1.5 for the northern, and 1.1 for the southern stars. We shall adopt an arbitrary criterion that if the value of $q$ is larger than those 

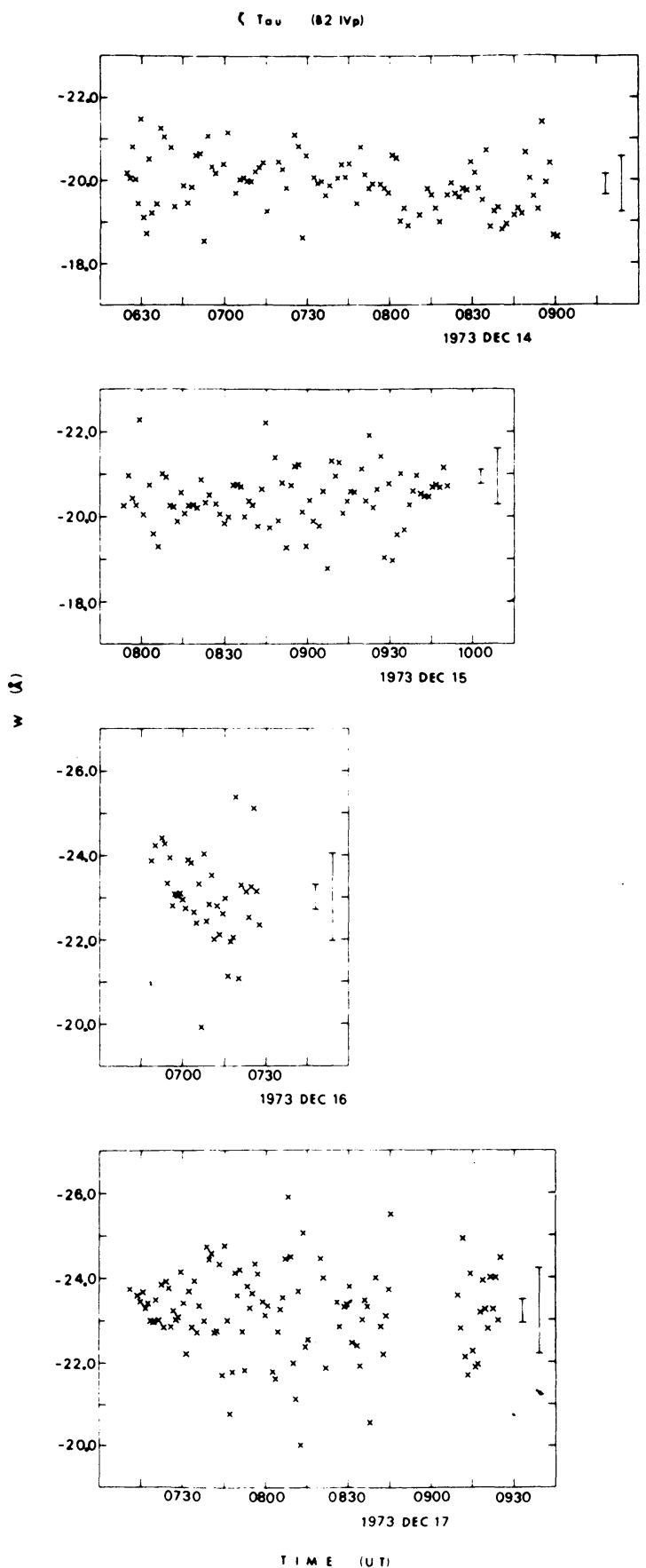

Fig. 2. H $\alpha$ equivalent width as a function of time for $\zeta$ Tau. The error bars are observed rms (larger) and the computed photon rms (smaller). 

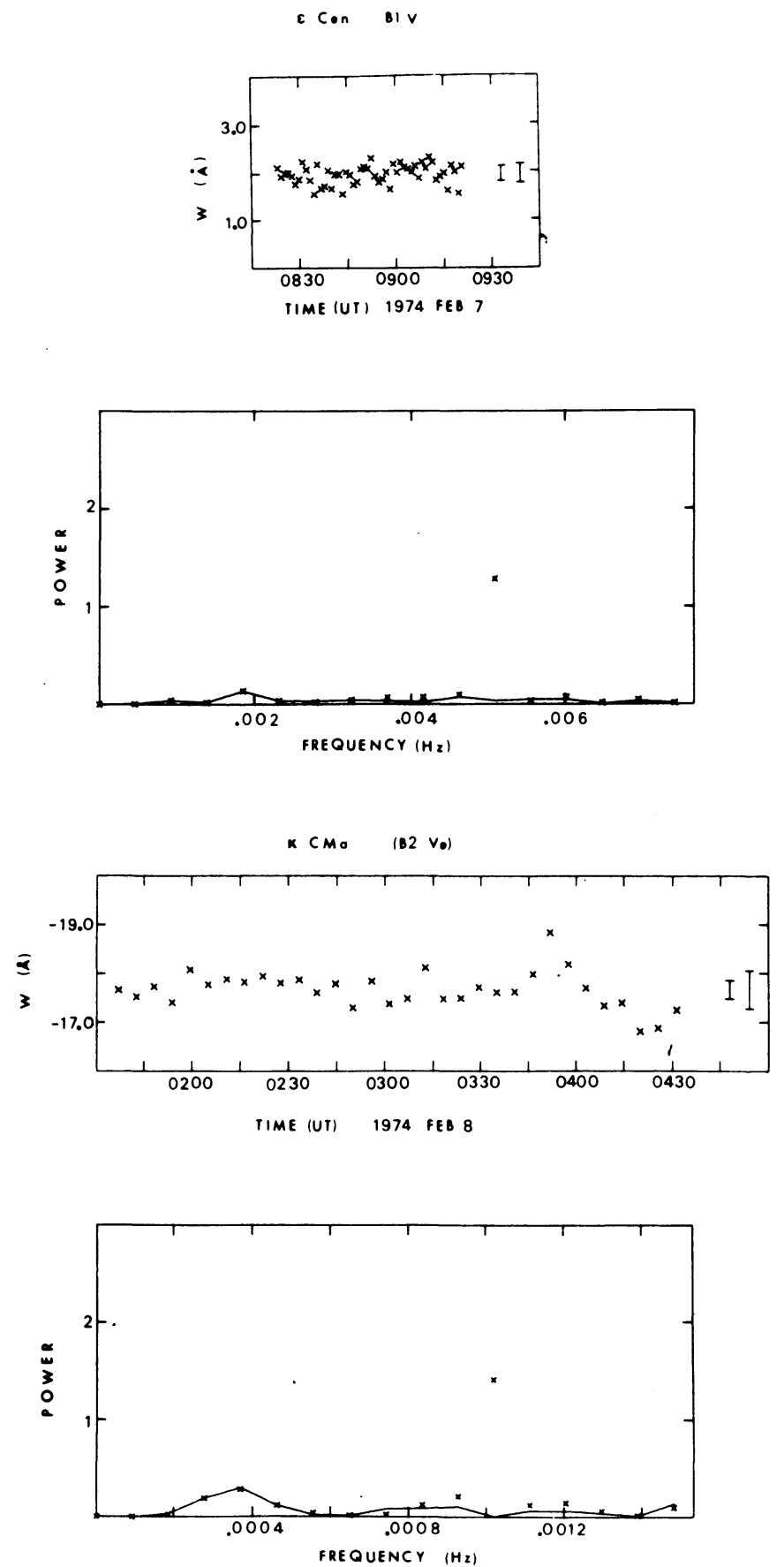

Fig. 3. H $\alpha$ equivalent width as a function of time, and the power spectra for $\varepsilon$ Cen and $\kappa \mathrm{CMa}$. The error bars are observed rms (larger) and the computed photon rms (smaller). In the power spectra, the crosses indicate power spectra with a pure sinusoidal variation of $0.5 \AA$ semi-amplitude superimposed on observed data. 

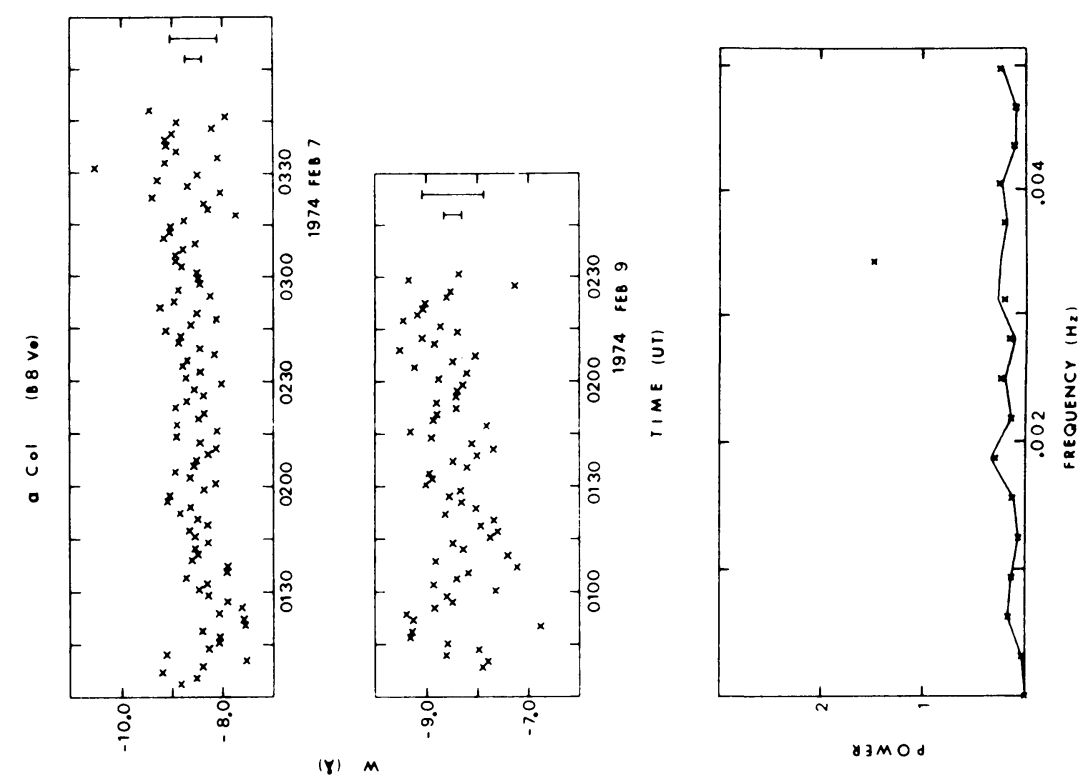

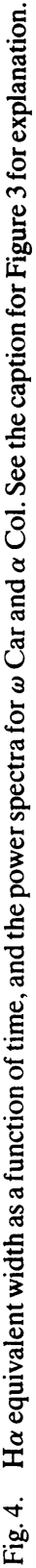
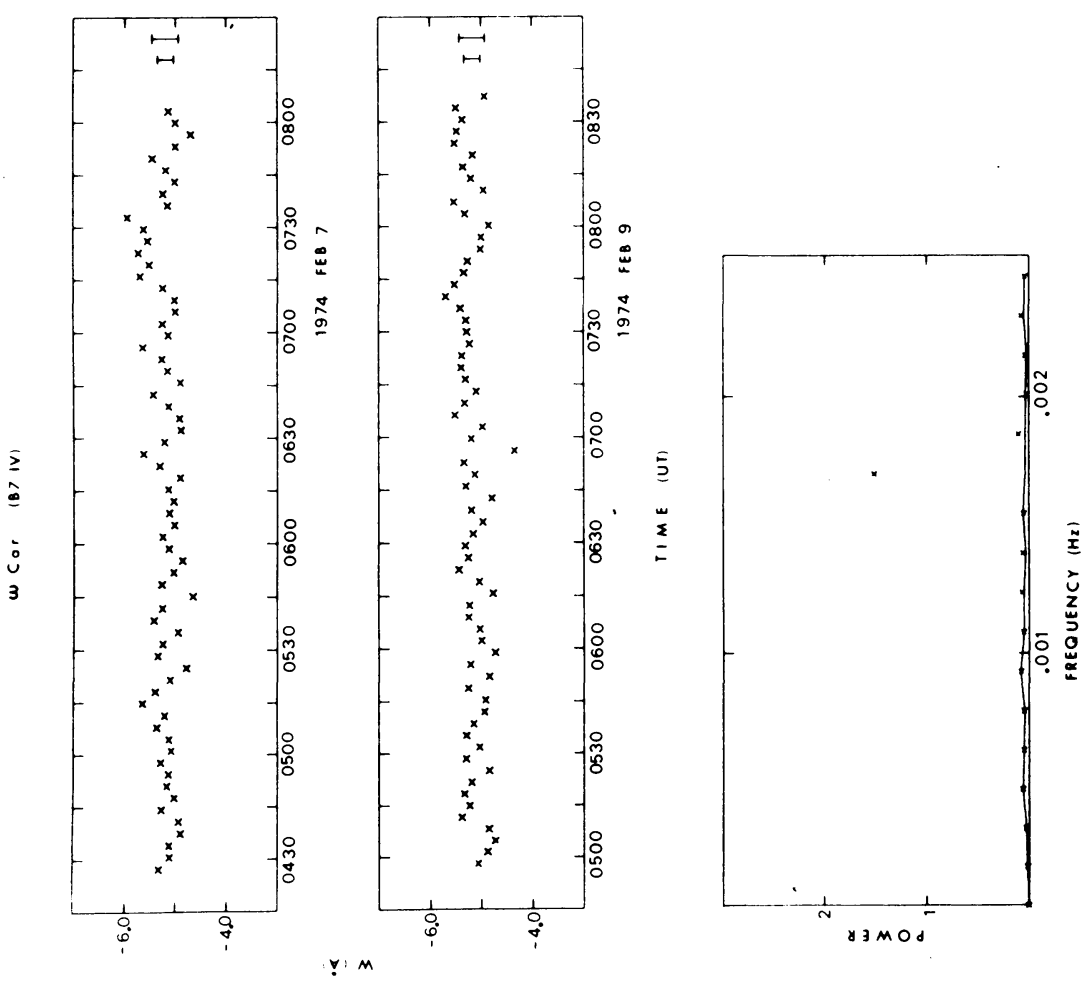

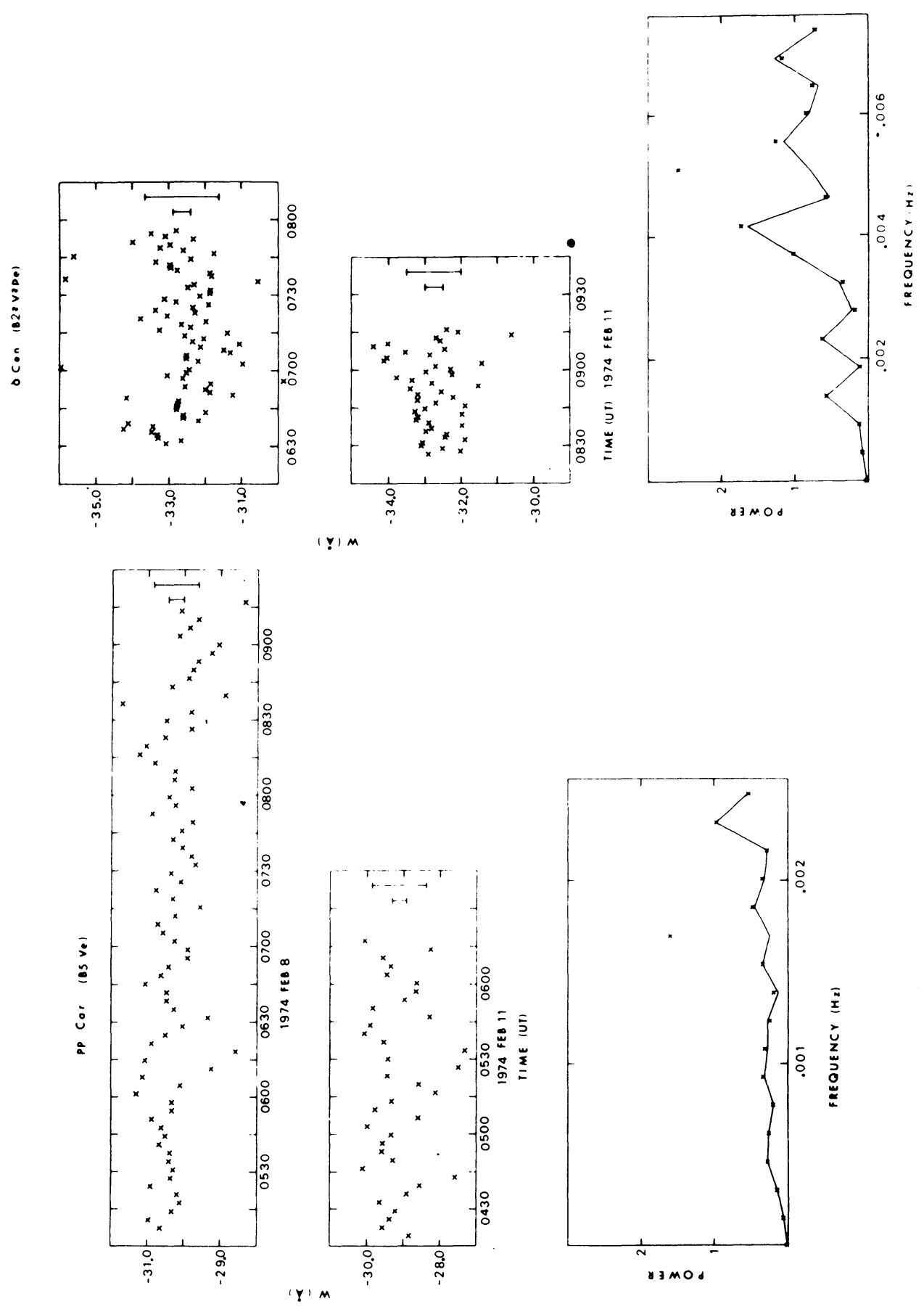
for the standard stars by more than a factor 2 , the variations are significant. On this basis, $\zeta$ Tau, $\alpha$ Col, PP Car, and $\delta$ Cen are judged to show significant variations in the emission strength of $\mathrm{H} \alpha$. In addition, for $\zeta$ Tau, there is a steady increase in the emission strength over four nights, from $W=-19.9$ to $-23.2 \AA$. The amplitudes of variations are a few percent (rms), with the shortest time scale being the time interval between two successive scans, on the order of 1-3 min. The mean power spectra show that there is no periodicity in any of these variations. In the remaining Be stars in these studies, the variations are either absent ( $\nu$ Gem and $\omega$ Car), or marginal (48 Per, $\kappa$ Dra, and $\kappa$ CMa).

The amount of variations found here is rather small, which makes it difficult to establish the reality of these rapid changes with absolute certainty. However, accumulated evidences indicate that there are short-term variations in the Balmer line emission strengths in some Be stars. The data are not sufficient to draw even preliminary conclusions regarding any characteristics which may be ascribed to those stars showing the variations. From the time scales involved, and the fact that the amplitudes of variations are small, it is evident that the variations are localized phenomena. Further observations of many more objects are required to answer the questions raised at the beginning of this paper.

\section{References}

Bahng, J. D. R.: 1971, Astrophys. J. 167, L75.

Bahng, J. D. R.: 1975, Monthly Notices Roy. Astron. Soc. 170, 611.

Delplace, A. M.: 1970, Astron. Astrophys. 7, 459.

Huang, S.-S.: 1972, Astrophys. J. 171, 549.

Huang, S.-S.: 1973, Astrophys. J. 183, 541.

Hutchings, J. B., Auman, J. R., Gower, A. C., and Walker, G. A. H.: 1971, Astrophys. J. 170 , L73.

McBeath, K. B.: 1974, 'Rapid Variations of Balmer Line Strengths in the Spectra of Be Stars', Northwestern University (Ph.D. Thesis).

Marlborough, J. M. and Zamir, M.: 1975, Astrophys. J. 195, 145.

Mihalas, D.: 1974, Astron. J. 79, 1111.

Prendergast, K. H. and Spiegel, E. A.: 1973, Comments Astrophys. Space Phys. (Part C) 5, 43.

Sanyal, A.: 1974, Bull. Am. Astron. Soc. 6, 460. 